\title{
Change Detection in Full and Dual Polarization, Single- and Multifrequency SAR Data
}

\author{
Nielsen, Allan Aasbjerg; Conradsen, Knut; Skriver, Henning
}

Published in:

I E E E Journal of Selected Topics in Applied Earth Observations and Remote Sensing

Link to article, DOI:

10.1109/JSTARS.2015.2416434

Publication date:

2015

Document Version

Peer reviewed version

Link back to DTU Orbit

Citation $(A P A)$ :

Nielsen, A. A., Conradsen, K., \& Skriver, H. (2015). Change Detection in Full and Dual Polarization, Single- and Multifrequency SAR Data. I E E E Journal of Selected Topics in Applied Earth Observations and Remote Sensing, 8(8), 4041-4048. https://doi.org/10.1109/JSTARS.2015.2416434

\section{General rights}

Copyright and moral rights for the publications made accessible in the public portal are retained by the authors and/or other copyright owners and it is a condition of accessing publications that users recognise and abide by the legal requirements associated with these rights.

- Users may download and print one copy of any publication from the public portal for the purpose of private study or research.

- You may not further distribute the material or use it for any profit-making activity or commercial gain

- You may freely distribute the URL identifying the publication in the public portal 


\title{
Change Detection in Full and Dual Polarization, Single- and Multi-Frequency SAR Data
}

\author{
Allan Aasbjerg Nielsen, Knut Conradsen, and Henning Skriver, Member, IEEE
}

\begin{abstract}
When the covariance matrix formulation is used for multi-look polarimetric synthetic aperture radar (SAR) data, the complex Wishart distribution applies. Based on this distribution a test statistic for equality of two complex variance-covariance matrices and an associated asymptotic probability of obtaining a smaller value of the test statistic are given. In a case study airborne EMISAR C- and L-band SAR images from the spring of 1998 covering agricultural fields and wooded areas near Foulum, Denmark, are used in single- and bi-frequency, bi-temporal change detection with full and dual polarimetry data.
\end{abstract}

Index Terms-Complex covariance matrix test statistic, complex Wishart distribution, quad polarization, full polarization, dual polarization, remote sensing change detection, EMISAR.

\section{INTRODUCTION}

$\mathbf{I}$ N 2003 we introduced a new test statistic for equality of two variance-covariance matrices following the complex Wishart distribution with an associated probability of observing a smaller value of the test statistic. We also demonstrated its use to change detection in both fully polarimetric and azimuthal symmetric SAR data, [1]. Full polarization is also known as quad polarization. An appendix gave details and mentioned the possibility of application of the test statistic and the associated probability to block-structured covariance SAR data. This feature was further described in [2] which also showed the application of the test statistic and the associated probability to edge detection in polarimetric SAR data. Application of the Hotelling-Lawley trace statistic to the change detection problem is described in [3].

In the early 2000s, not many workers had access to polarimetric SAR data. With the advent of several spaceborne polarimetric SAR instruments this situation has improved. These instruments include

- the Japanese ALOS (a.k.a. DAICHI), L-band with single, dual and full polarization,

- the Canadian Radarsat-2, C-band with single, dual and full polarization,

- the German TerraSAR-X, X-band with single, dual and full polarization,

- the Italian COSMO-SkyMed, X-band with single and dual polarization,

A. A. Nielsen and K. Conradsen are with DTU Compute, the Department of Applied Mathematics and Computer Science, H. Skriver is with DTU Space, the National Space Institute. Both departments are with the Technical University of Denmark, DK-2800 Kgs. Lyngby, Denmark. Correspondence to AAN, e-mail alan@dtu.dk, internet http://people.compute.dtu.dk/alan. The Danish National Research Councils partly under the Earth Observation Programme and partly under the ESA Follow-on Research Programme funded part of the work reported here. The EMISAR data acquisitions and part of the data processing have been funded by the Danish National Research Foundation.
- the European (ESA) Sentinel-1, C-band with single and dual polarization.

For power supply and coverage reasons some of the instruments among the above which have full polarization capability are often operated in (either single or) dual polarization modes, i.e., they transmit one polarization only and receive (either one or) both polarizations. We therefore think that a revisit to the methods described and sketched in [1], [2] with emphasis on dual polarization and multi-frequency SAR data is timely.

Section II briefly recapitulates the fundamental results with some focus on dual polarization and multi-frequency data, Section III gives a case study with single- and multi-frequency, full and dual polarimetry data, Section IV discusses the results, Section V briefly mentions Matlab, ENVI/IDL and Python code to carry out the analysis, and Section VI gives conclusions and directions for future work.

\section{THEORY}

\section{A. Synthetic Aperture Radar}

A fully polarimetric SAR measures the 2 by 2 complex socalled scattering matrix at each resolution cell on the ground. The scattering matrix relates the incident and the scattered electric fields, [4]. If $S_{r t}$ denotes the complex scattering amplitude for receive and transmit polarization $(r, t \in\{h, v\}$ for horizontal and vertical polarization), then reciprocity, which normally applies to natural targets, gives $S_{h v}=S_{v h}$ (in the backscattering direction using the backscattering alignment convention) [4]. Assuming reciprocity, the scattering matrix is represented by the three-component complex target vector $s=\left[\begin{array}{lll}S_{h h} & S_{h v} & S_{v v}\end{array}\right]^{T},{ }^{T}$ means transpose.

The inherent speckle in the SAR data can be reduced by spatial averaging at the expense of spatial resolution. In this so-called multi-look case a more appropriate representation of the backscattered signal is the covariance matrix in which the average properties of a group of resolution cells can be expressed in a single matrix formed by the outer products of the averaged target vectors. The average covariance matrix is defined as [4]

$$
\langle\boldsymbol{C}\rangle_{f u l l}=\left[\begin{array}{lll}
\left\langle S_{h h} S_{h h}^{*}\right\rangle & \left\langle S_{h h} S_{h v}^{*}\right\rangle & \left\langle S_{h h} S_{v v}^{*}\right\rangle \\
\left\langle S_{h v} S_{h h}^{*}\right\rangle & \left\langle S_{h v} S_{h v}^{*}\right\rangle & \left\langle S_{h v} S_{v v}^{*}\right\rangle \\
\left\langle S_{v v} S_{h h}^{*}\right\rangle & \left\langle S_{v v} S_{h v}^{*}\right\rangle & \left\langle S_{v v} S_{v v}^{*}\right\rangle
\end{array}\right]
$$

where $\langle\cdot\rangle$ denotes ensemble averaging and ${ }^{*}$ denotes complex conjugation. Reciprocity results in a covariance matrix (Equation 1) with rank 3. The components of the covariance matrix containing both co- and cross-polarized scattering matrix elements $\left(\left\langle S_{h h} S_{h v}^{*}\right\rangle\right.$ and $\left.\left\langle S_{h v} S_{v v}^{*}\right\rangle\right)$ often contain little 
information. For randomly distributed targets with azimuthal symmetry the elements are zero [5] resulting in the covariance matrix

$$
\langle\boldsymbol{C}\rangle_{a z i m}=\left[\begin{array}{ccc}
\left\langle S_{h h} S_{h h}^{*}\right\rangle & 0 & \left\langle S_{h h} S_{v v}^{*}\right\rangle \\
0 & \left\langle S_{h v} S_{h v}^{*}\right\rangle & 0 \\
\left\langle S_{v v} S_{h h}^{*}\right\rangle & 0 & \left\langle S_{v v} S_{v v}^{*}\right\rangle
\end{array}\right]
$$

Spaceborne instruments often transmit only one polarization, say horizontal, and receive both polarizations giving rise to dual polarization data. In this case we have the components $\left\langle S_{h h} S_{h h}^{*}\right\rangle,\left\langle S_{h h} S_{h v}^{*}\right\rangle$ and $\left\langle S_{h v} S_{h v}^{*}\right\rangle$ only (which is a little unfortunate since the complex terms we retain are the ones we force to zero in the azimuthal symmetry case). The resulting covariance matrix

$$
\langle\boldsymbol{C}\rangle_{\text {dual }}=\left[\begin{array}{ll}
\left\langle S_{h h} S_{h h}^{*}\right\rangle & \left\langle S_{h h} S_{h v}^{*}\right\rangle \\
\left\langle S_{h v} S_{h h}^{*}\right\rangle & \left\langle S_{h v} S_{h v}^{*}\right\rangle
\end{array}\right]
$$

has rank 2. (We could also transmit vertical polarization only and receive both or transmit and receive both.)

For both full and dual polarimetry the case where we have the diagonal elements of the covariance matrix only is also relevant.

\section{B. Test for Equality of Two Complex Covariance Matrices}

If the independent $p$ by $p$ Hermitian, positive definite matrices $\boldsymbol{X}$ and $\boldsymbol{Y}$ are complex Wishart distributed $\left(\boldsymbol{X}=n\langle\boldsymbol{C}\rangle_{x}\right.$ and $\boldsymbol{Y}=m\langle\boldsymbol{C}\rangle_{y}, n$ and $m$ are the number of looks), i.e., $\boldsymbol{X} \in W_{C}\left(p, n, \boldsymbol{\Sigma}_{x}\right)$ with $\hat{\boldsymbol{\Sigma}}_{x}=\frac{1}{n} \boldsymbol{X}$ and $\boldsymbol{Y} \in W_{C}\left(p, m, \boldsymbol{\Sigma}_{y}\right)$ with $\hat{\boldsymbol{\Sigma}}_{y}=\frac{1}{m} \boldsymbol{Y}$, then the statistic to test the so-called null hypothesis $H_{0}$ for equality of the two complex Wishart matrices

$$
H_{0}: \boldsymbol{\Sigma}_{x}=\boldsymbol{\Sigma}_{y}
$$

against all alternatives, is (see [1]; for the real case see [6])

$$
Q=\frac{(n+m)^{p(n+m)}}{n^{p n} m^{p m}} \frac{|\boldsymbol{X}|^{n}|\boldsymbol{Y}|^{m}}{|\boldsymbol{X}+\boldsymbol{Y}|^{n+m}}
$$

where $|\cdot|$ denotes the determinant; $Q \in[0,1]$ with $Q=1$ for equality. For the logarithm of $Q$ we get

$$
\begin{aligned}
\ln Q= & p[(n+m) \ln (n+m)-n \ln (n)-m \ln (m)] \\
& +n \ln |\boldsymbol{X}|+m \ln |\boldsymbol{Y}|-(n+m) \ln |\boldsymbol{X}+\boldsymbol{Y}| .
\end{aligned}
$$

If $n=m$ which is often the case we get

$$
\ln Q=n(2 p \ln 2+\ln |\boldsymbol{X}|+\ln |\boldsymbol{Y}|-2 \ln |\boldsymbol{X}+\boldsymbol{Y}|) .
$$

If

$$
\rho=1-\frac{2 p^{2}-1}{6 p}\left(\frac{1}{n}+\frac{1}{m}-\frac{1}{n+m}\right)
$$

and

$$
\begin{aligned}
\omega_{2}= & -\frac{p^{2}}{4}\left(1-\frac{1}{\rho}\right)^{2}+\frac{p^{2}\left(p^{2}-1\right)}{24 \rho^{2}} \\
& \cdot\left(\frac{1}{n^{2}}+\frac{1}{m^{2}}-\frac{1}{(n+m)^{2}}\right)
\end{aligned}
$$

and if the observed value of $-2 \rho \ln Q$ is $z=-2 \rho \ln q_{\mathrm{obs}}$, then the probability of finding a smaller value of $-2 \rho \ln Q$ is

$$
\begin{aligned}
& P\{-2 \rho \ln Q \leq z\} \simeq P\left\{\chi^{2}\left(p^{2}\right) \leq z\right\} \\
& \quad+\omega_{2}\left[P\left\{\chi^{2}\left(p^{2}+4\right) \leq z\right\}-P\left\{\chi^{2}\left(p^{2}\right) \leq z\right\}\right] .
\end{aligned}
$$

$1-P\{-2 \rho \ln Q \leq z\}=P\left\{Q<q_{\text {obs }}\right\}$ is the probability of equality, i.e., the probability of no change.

For full polarimetry data $p=3$, for dual polarimetry $p=2$, and for $\mathrm{HH}, \mathrm{HV}$ or $\mathrm{VV}$ data $p=1$. In the latter case $\boldsymbol{X}$ and $\boldsymbol{Y}$ are therefore scalars $X$ and $Y$, and $Q$ reduces to

$$
Q=\frac{(n+m)^{n+m}}{n^{n} m^{m}} \frac{X^{n} Y^{m}}{(X+Y)^{n+m}}
$$

which is equivalent to the well-known likelihood ratio test statistic for the equality of two gamma parameters [1], [7], [8]. In this case the test statistic $(m Y) /(n X)$ follows an $F(2 m, 2 n)$-distribution.

The expression in Equation 5 is a second order series expansion ( $\rho$ is chosen so that the coefficient of the first term, $\omega_{1}$, is zero) and studies show that inclusion of more terms improves the approximation only very little, especially for number of looks $n$ and $m>\sim 10$ [9].

1) Azimuthal Symmetry: The products between the copolarized and the cross-polarized terms (i.e., the absolute values of $S_{h h} S_{h v}^{*}$ and $S_{h v} S_{v v}^{*}$ ) often contain little information, and for the azimuthal symmetry case they are set to zero. In this case, by swapping first rows and then columns two and three in $\langle\boldsymbol{C}\rangle_{a z i m}$ in Equation 2 we obtain

$$
\left[\begin{array}{ccc}
\left\langle S_{h h} S_{h h}^{*}\right\rangle & \left\langle S_{h h} S_{v v}^{*}\right\rangle & 0 \\
\left\langle S_{v v} S_{h h}^{*}\right\rangle & \left\langle S_{v v} S_{v v}^{*}\right\rangle & 0 \\
0 & 0 & \left\langle S_{h v} S_{h v}^{*}\right\rangle
\end{array}\right]=\left[\begin{array}{cc}
\boldsymbol{X}_{1} & 0 \\
0 & \boldsymbol{X}_{2}
\end{array}\right],
$$

where $\boldsymbol{X}_{1}$ is $p_{1}$ by $p_{1}$ (here 2 by 2 ) and $\boldsymbol{X}_{2}=\left\langle S_{h v} S_{h v}^{*}\right\rangle$ is $p_{2}$ by $p_{2}$ (here 1 by 1 ). This matrix multiplied by $n$ is not Wishart distributed. However, as described in [1] we arrive at the same test statistic as in Equation 4 with $p=p_{1}+p_{2}$. If

$$
\begin{aligned}
f_{1} & =p_{1}^{2} \\
f_{2} & =p_{2}^{2} \\
f & =f_{1}+f_{2} \\
\rho_{1} & =1-\frac{2 f_{1}-1}{6 p_{1}}\left(\frac{1}{n}+\frac{1}{m}-\frac{1}{n+m}\right) \\
\rho_{2} & =1-\frac{2 f_{2}-1}{6 p_{2}}\left(\frac{1}{n}+\frac{1}{m}-\frac{1}{n+m}\right) \\
\rho & =\frac{1}{f}\left(f_{1} \rho_{1}+f_{2} \rho_{2}\right)
\end{aligned}
$$

and

$$
\begin{aligned}
\omega_{2}= & -\frac{f}{4}\left(1-\frac{1}{\rho}\right)^{2}+\frac{f_{1}\left(f_{1}-1\right)+f_{2}\left(f_{2}-1\right)}{24 \rho^{2}} \\
& \cdot\left(\frac{1}{n^{2}}+\frac{1}{m^{2}}-\frac{1}{(n+m)^{2}}\right)
\end{aligned}
$$

then the probability of finding a smaller value of $-2 \rho \ln Q$ is

$$
\begin{aligned}
& P\{-2 \rho \ln Q \leq z\} \simeq P\left\{\chi^{2}(f) \leq z\right\} \\
& \quad+\omega_{2}\left[P\left\{\chi^{2}(f+4) \leq z\right\}-P\left\{\chi^{2}(f) \leq z\right\}\right] .
\end{aligned}
$$

i.e., the same as in Equation 5 but this time with different values of $f, \rho$ and $\omega_{2}$. 
2) Diagonal Only: The diagonal only case for both full and dual polarization may be handled in a fashion similar to the block structure described in the previous section, see also [1].

3) Other Block Diagonal Cases: Multi-frequency polarimetric data can also be described using the complex Wishart distribution. If $k$ is the number of polarimetric datasets acquired at different frequencies (and/or acquisition time points), and if the target vector data are available, the complex target vector is simply redefined as $s=\left[\begin{array}{llll}\boldsymbol{s}_{1}^{T} & \ldots & \boldsymbol{s}_{k}^{T}\end{array}\right]^{T}$ resulting in the average covariance matrix

$$
\langle\boldsymbol{C}\rangle=\left[\begin{array}{ccc}
\langle\boldsymbol{C}\rangle_{1} & \cdots & \langle\boldsymbol{C}\rangle_{1 k} \\
\vdots & \ddots & \vdots \\
\langle\boldsymbol{C}\rangle_{k 1} & \cdots & \langle\boldsymbol{C}\rangle_{k}
\end{array}\right]
$$

where $\langle\boldsymbol{C}\rangle_{i}$ is the average covariance matrix for acquisition $i$, and the elements of the complex matrices $\langle\boldsymbol{C}\rangle_{i j}, i \neq j$, depend on the correlation between the acquisitions $\left(\langle\boldsymbol{C}\rangle_{i j}=\right.$ $\langle\boldsymbol{C}\rangle_{j i}^{*}$. Again, the $p_{1}+\cdots+p_{k}$ by $p_{1}+\cdots+p_{k}$ Hermitian matrix $n\langle\boldsymbol{C}\rangle$ follows a complex Wishart distribution (where $n$ is number of looks). Often, however, the polarimetric SAR data from the acquisitions are already in the covariance matrix representation. In this case, the matrices $\langle\boldsymbol{C}\rangle_{i j}$ cannot be formed, and $n\langle\boldsymbol{C}\rangle$ does not follow a complex Wishart distribution, as some of the elements of the covariance matrix have been set to zero and we obtain a block diagonal $\langle\boldsymbol{C}\rangle$

$$
\langle\boldsymbol{C}\rangle=\left[\begin{array}{ccc}
\langle\boldsymbol{C}\rangle_{1} & \cdots & \mathbf{0} \\
\vdots & \ddots & \vdots \\
\mathbf{0} & \cdots & \langle\boldsymbol{C}\rangle_{k}
\end{array}\right] .
$$

In this case we get the same test statistic as in the above cases (Equation 4) with $p=p_{1}+\cdots+p_{k}$. If

$$
\begin{aligned}
f_{i} & =p_{i}^{2} \\
f & =f_{1}+\cdots+f_{k} \\
\rho_{i} & =1-\frac{2 f_{i}-1}{6 p_{i}}\left(\frac{1}{n}+\frac{1}{m}-\frac{1}{n+m}\right) \\
\rho & =\frac{1}{f}\left(f_{1} \rho_{1}+\cdots+f_{k} \rho_{k}\right)
\end{aligned}
$$

and

$$
\begin{aligned}
\omega_{2}= & -\frac{f}{4}\left(1-\frac{1}{\rho}\right)^{2} \\
& +\frac{f_{1}\left(f_{1}-1\right)+\cdots+f_{k}\left(f_{k}-1\right)}{24 \rho^{2}} \\
& \cdot\left(\frac{1}{n^{2}}+\frac{1}{m^{2}}-\frac{1}{(n+m)^{2}}\right)
\end{aligned}
$$

then the probability of finding a smaller value of $-2 \rho \ln Q$ is the same as in Equation 5 with the immediately above values of $f, \rho$ and $\omega_{2}$.

\section{Practical Issues}

Here we deal with some computer implementation issues, namely the calculation of the determinants involved, and the calculation of probabilities. We also give examples on the handling of multi-frequency data based on the test statistic in Equation 4 and the probability measure in Equation 5 with associated values of $f, \rho$ and $\omega_{2}$.
1) Determinants: Let us write $\boldsymbol{Z}=n\langle\boldsymbol{C}\rangle$ as ( $n$ is the number of looks)

$$
\begin{aligned}
\boldsymbol{Z} & =n\left[\begin{array}{lll}
\left\langle S_{h h} S_{h h}^{*}\right\rangle & \left\langle S_{h h} S_{h v}^{*}\right\rangle & \left\langle S_{h h} S_{v v}^{*}\right\rangle \\
\left\langle S_{h v} S_{h h}^{*}\right\rangle & \left\langle S_{h v} S_{h v}^{*}\right\rangle & \left\langle S_{h v} S_{v v}^{*}\right\rangle \\
\left\langle S_{v v} S_{h h}^{*}\right\rangle & \left\langle S_{v v} S_{h v}^{*}\right\rangle & \left\langle S_{v v} S_{v v}^{*}\right\rangle
\end{array}\right] \\
& =\left[\begin{array}{ccc}
k & a & \rho \\
a^{*} & \xi & b \\
\rho^{*} & b^{*} & \zeta
\end{array}\right] .
\end{aligned}
$$

This gives the determinant

$$
|\boldsymbol{Z}|=k \xi \zeta+a b \rho^{*}+\rho a^{*} b^{*}-|\rho|^{2} \xi-|b|^{2} k-|a|^{2} \zeta .
$$

The first, fourth, fifth and sixth terms are real. The second and third terms are complex and each others conjugate

$$
\begin{aligned}
a b \rho^{*}= & \left(a_{R}+i a_{I}\right)\left(b_{R}+i b_{I}\right)\left(\rho_{R}-i \rho_{I}\right) \\
= & \rho_{R}\left(a_{R} b_{R}-a_{I} b_{I}\right)+\rho_{I}\left(a_{I} b_{R}+a_{R} b_{I}\right) \\
& +i\left[\rho_{R}\left(a_{I} b_{R}+a_{R} b_{I}\right)-\rho_{I}\left(a_{R} b_{R}-a_{I} b_{I}\right)\right] \\
a^{*} b^{*} \rho= & \left(a_{R}-i a_{I}\right)\left(b_{R}-i b_{I}\right)\left(\rho_{R}+i \rho_{I}\right) \\
= & \rho_{R}\left(a_{R} b_{R}-a_{I} b_{I}\right)+\rho_{I}\left(a_{I} b_{R}+a_{R} b_{I}\right) \\
& -i\left[\rho_{R}\left(a_{I} b_{R}+a_{R} b_{I}\right)-\rho_{I}\left(a_{R} b_{R}-a_{I} b_{I}\right)\right] .
\end{aligned}
$$

Since the imaginary parts cancel out, the sum of the two terms is real and equal to twice the real part.

2) Probabilities: Rewriting the approximate probability of finding a smaller value of $-2 \rho \ln Q$ in Equation 5, see also [1], [2], the probabilities involved can be found by two calls to the incomplete gamma function, $\gamma\left(\frac{\nu}{2}, \frac{X^{2}}{2}\right)$ [10], [11] where $\nu$ is the number of degrees of freedom and $X^{2}$ is the test statistic

$$
\begin{aligned}
P\{-2 \rho \ln Q \leq z\} \simeq & {\left[1-\omega_{2}\right] \gamma\left(\frac{f}{2},-\rho \ln Q\right) } \\
& +\omega_{2} \gamma\left(\frac{f}{2}+2,-\rho \ln Q\right) .
\end{aligned}
$$

Experiments show that for this probability measure to stay nonnegative, for full polarimetry $(p=3)$ the number of looks must be no smaller than 2.274. For dual polarimetry $(p=2)$ the number is 1.206 .

3) Multi-Frequency Data: Let us consider for example fully polarized $\mathrm{C}$ - and L-band data given as covariance data, i.e., we do not have access to the complex target vectors themselves, only their outer products. Here we can form the joint covariance matrix multiplied by $n$, see Equations 1, 6 and 7 ,

$$
\boldsymbol{Z}=\left[\begin{array}{cccccc}
k_{C} & a_{C} & \rho_{C} & 0 & 0 & 0 \\
a_{C}^{*} & \xi_{C} & b_{C} & 0 & 0 & 0 \\
\rho_{C}^{*} & b_{C}^{*} & \zeta_{C} & 0 & 0 & 0 \\
0 & 0 & 0 & k_{L} & a_{L} & \rho_{L} \\
0 & 0 & 0 & a_{L}^{*} & \xi_{L} & b_{L} \\
0 & 0 & 0 & \rho_{L}^{*} & b_{L}^{*} & \zeta_{L}
\end{array}\right] .
$$

In this case $k=2, p_{1}=p_{2}=3$ and $p=6$. For number of looks equal to 13 (as in the examples below) $\rho=0.8910$ and $\omega_{2}=0.0109$. The determinant equals the product of the two determinants of each block matrix, see Equation 8. 


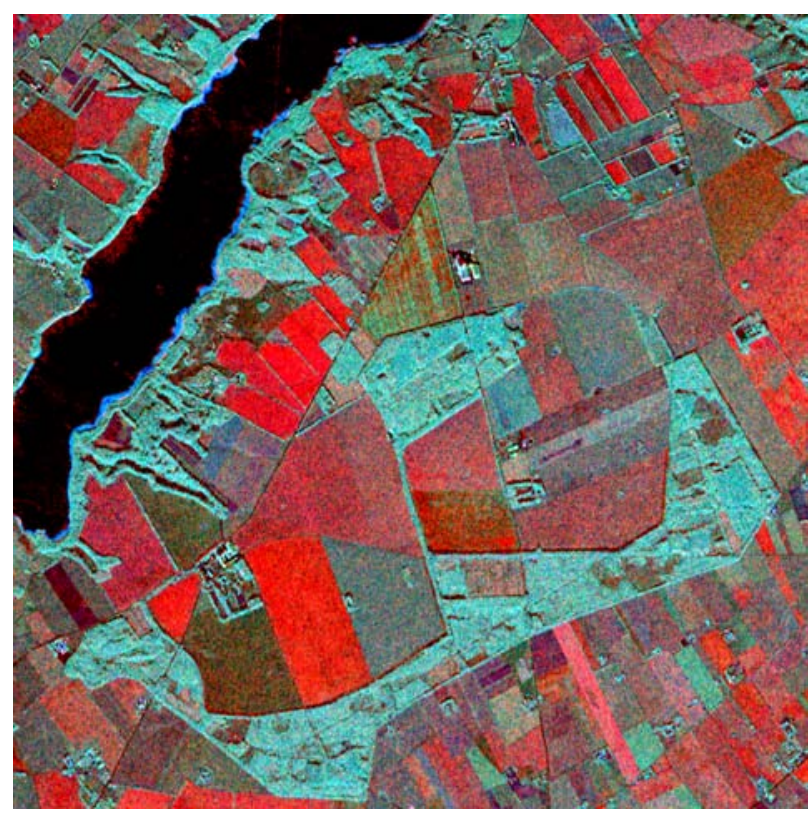

Fig. 1. EMISAR C-band data C64 20 May 1998, Pauli RGB.

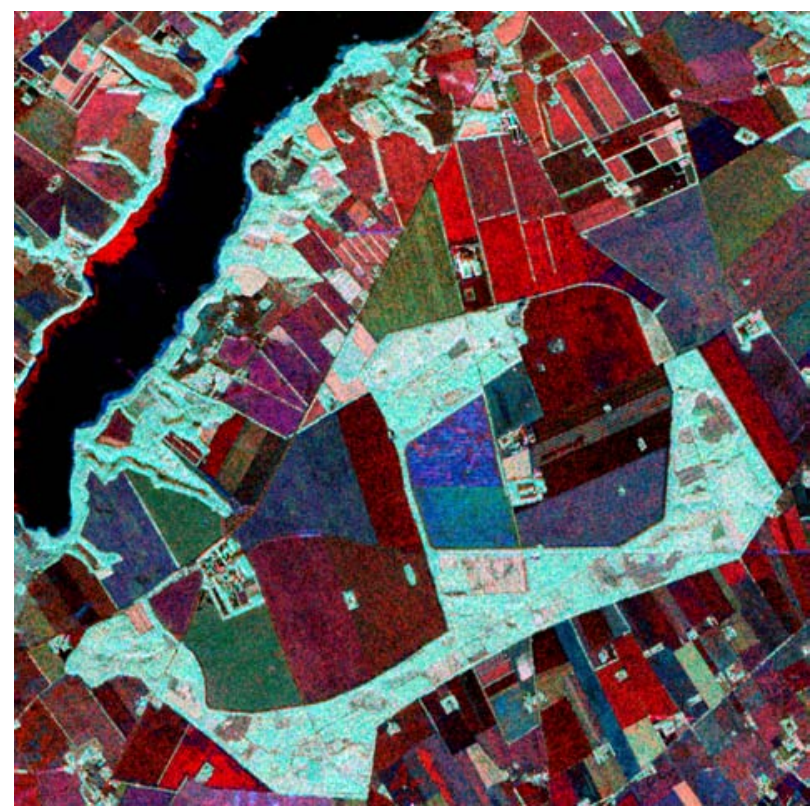

Fig. 2. EMISAR L-band data L64 20 May 1998, Pauli RGB.

In the same situation for dual polarization C- and L-band data, say, $\mathrm{HH}$ and $\mathrm{HV}$, we get the joint covariance matrix multiplied by $n$, see Equations 3, 6 and 7,

$$
\boldsymbol{Z}=\left[\begin{array}{cccc}
k_{C} & a_{C} & 0 & 0 \\
a_{C}^{*} & \xi_{C} & 0 & 0 \\
0 & 0 & k_{L} & a_{L} \\
0 & 0 & a_{L}^{*} & \xi_{L}
\end{array}\right]
$$

In this case $k=2, p_{1}=p_{2}=2$ and $p=4$. For number of looks equal to 13 (as in the examples below) $\rho=0.9327$ and $\omega_{2}=0.0015$. Again the determinant equals the product, here

$$
|\boldsymbol{Z}|=\left(k_{C} \xi_{C}-\left|a_{C}\right|^{2}\right)\left(k_{L} \xi_{L}-\left|a_{L}\right|^{2}\right) .
$$

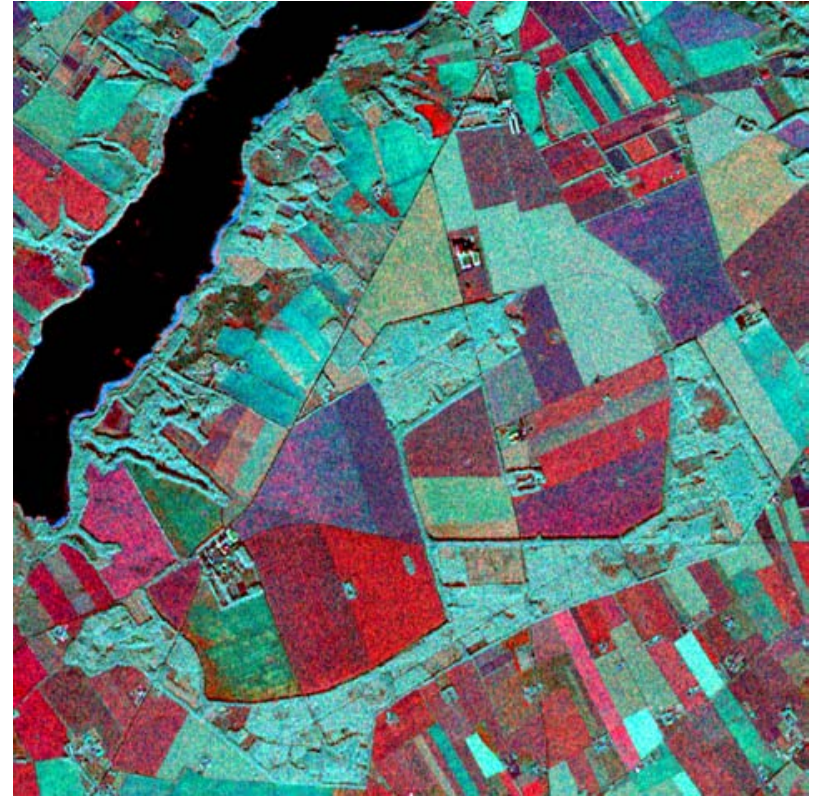

Fig. 3. EMISAR C-band data C65 16 June 1998, Pauli RGB.

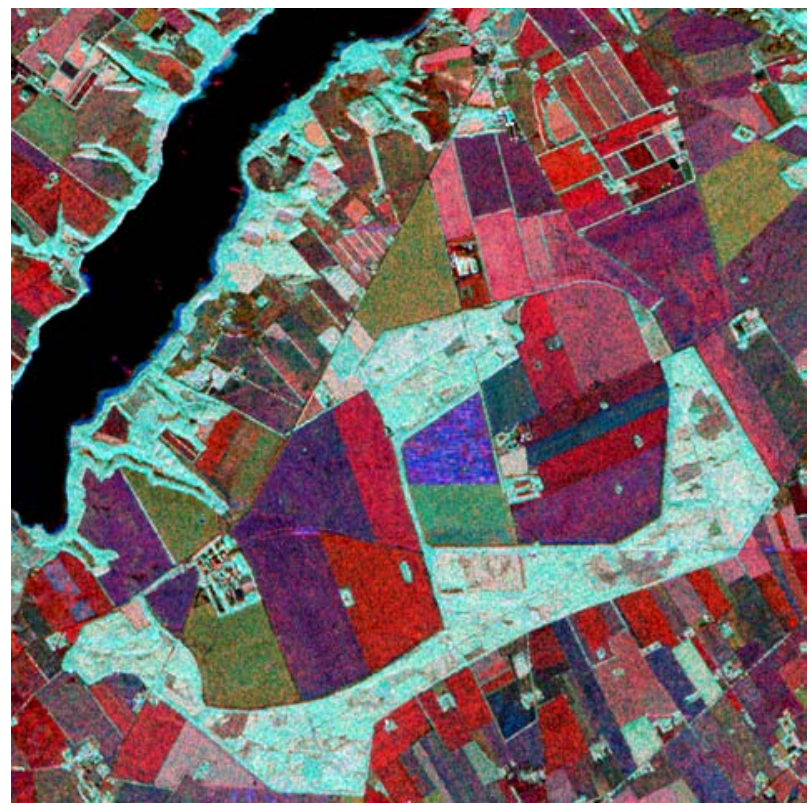

Fig. 4. EMISAR L-band data L65 16 June 1998, Pauli RGB.

\section{CAse Studies}

Full polarimetry, C- and L-band EMISAR [12], [13] data from the following dates

- 20 May 1998 (called C64 and L64),

- 16 June 1998 (called C65 and L65),

are used. The images have 5 meter pixels, 1024 lines and 1024 samples. Number of looks is 13 ( $n$ and $m$ above).

Figures 1 and 2 show RGB combinations of the diagonal elements, i.e., the backscatter coefficients, of the full polarimetry covariance matrix for C64 and L64. Figures 3 and 4 show RGB combinations of the diagonal elements (again, the backscatter coefficients) of the full polarimetry covariance matrix for C65 


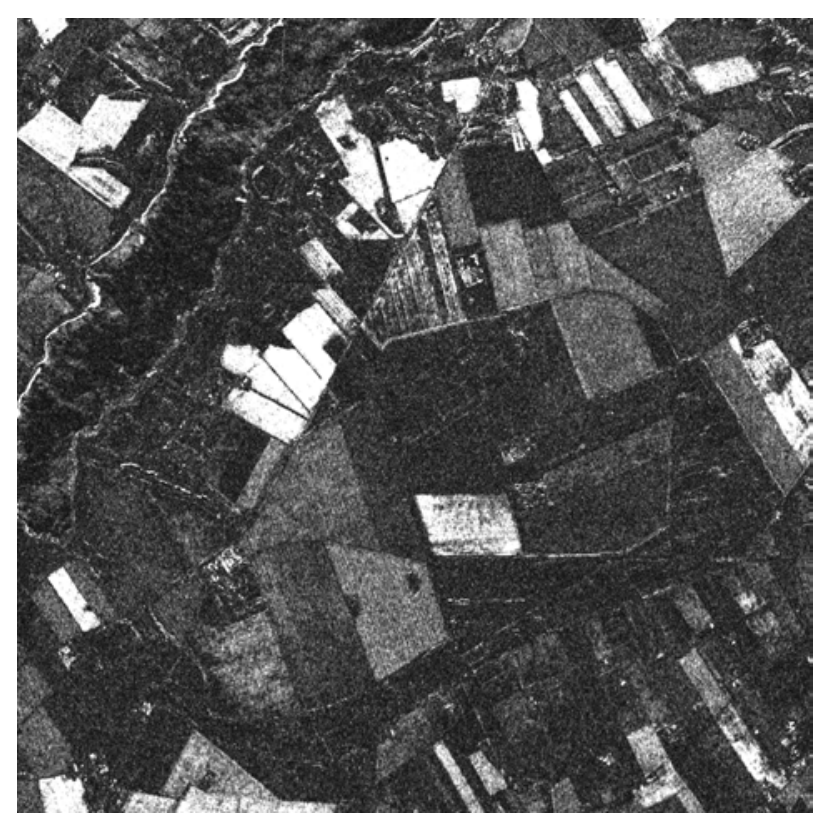

Fig. 5. $\quad-2 \rho \ln Q$ for C64 vs C65, full polarimetry.

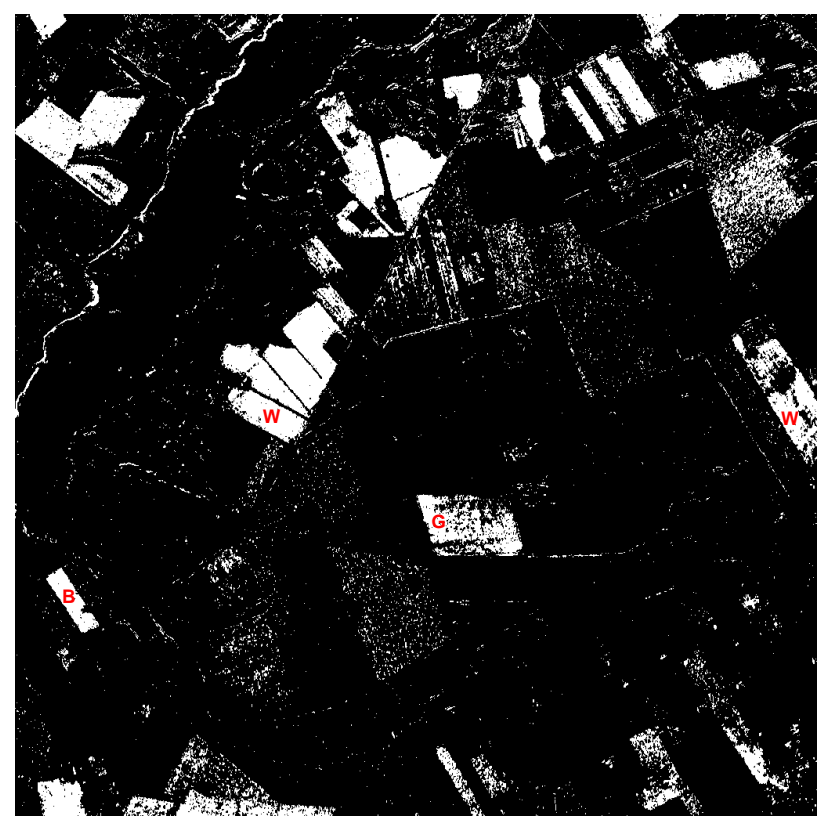

Fig. 6. $P\{-2 \rho \ln Q \leq z\}$ thresholded at 0.9999 for C64 vs C65, full polarimetry. "B" marks a beets field, "W" marks winter barley fields. "G" marks a grass field.

and L65. The two C-band images are stretched identically and so are the two L-band images.

\section{A. Bi-Temporal Multi-Frequency Change Detection, Full Po- larimetry}

Here we use the data C64/L64 and C65/L65 in a joint analysis as sketched in Section II-C3 to test for change in the bi-frequency, bi-temporal data.

First, Figures 5 and 7 show $-2 \rho \ln Q$ for C-band and Lband alone (stretched between 0 and 50). Figures 6 and 8 show the corresponding probabilities thresholded at 0.9999 . In

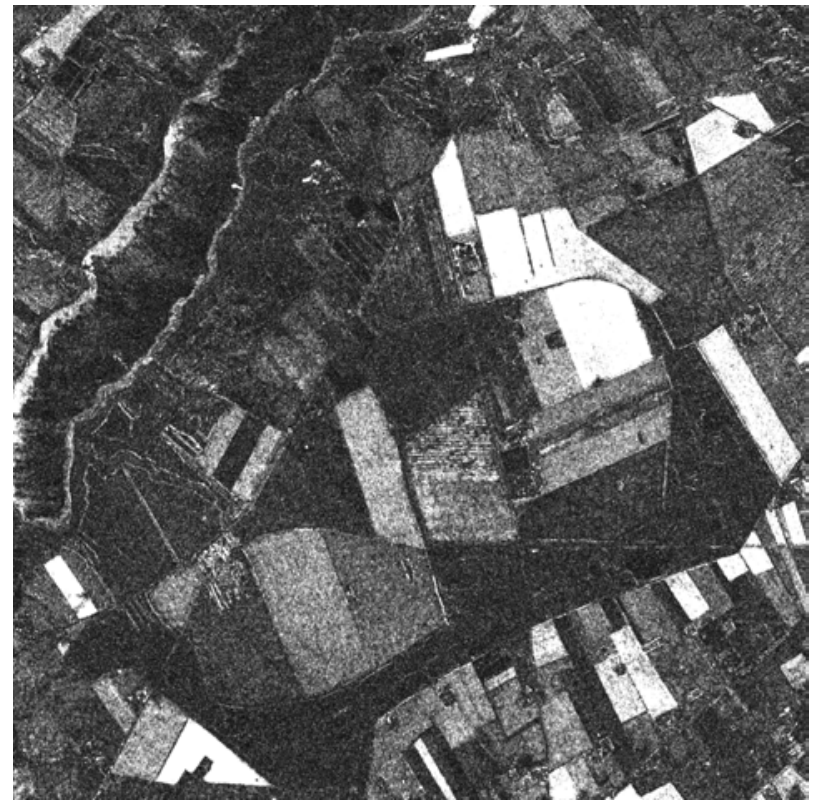

Fig. 7. $-2 \rho \ln Q$ for L64 vs L65, full polarimetry.

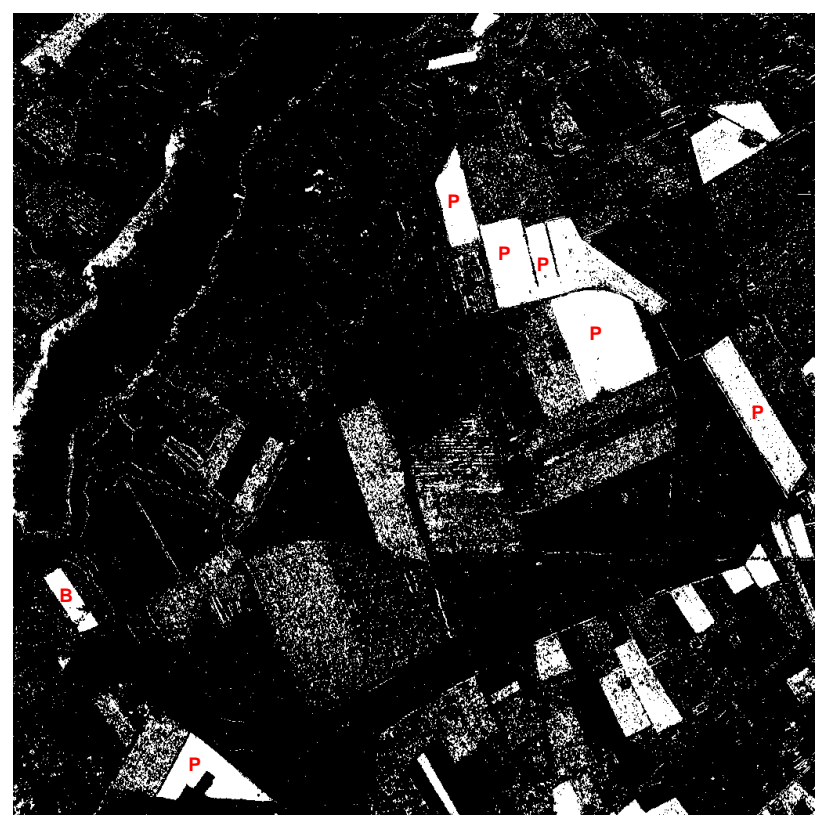

Fig. 8. $P\{-2 \rho \ln Q \leq z\}$ thresholded at 0.9999 for L64 vs L65, full polarimetry. "B" marks a beets field, "P" marks pea fields.

Figures 6 and 8 "B" marks a beets field, "P" marks pea fields, "W" winter barley fields, and "G" a grass field. We have no in situ information on the other highlighted but unmarked fields in the images.

Figures 9 and 10 show $-2 \rho \ln Q$ (stretched between 0 and 100 ) and $P$ (the probability of finding a smaller value of $-2 \rho \ln Q$ ) thresholded at 0.9999 for full polarimetry C64/L64 vs C65/L65.

\section{B. Bi-Temporal Change Detection, Dual Polarimetry}

As an example we here use the HH/HV data L64 and L65 to test for change in the bi-temporal data. 


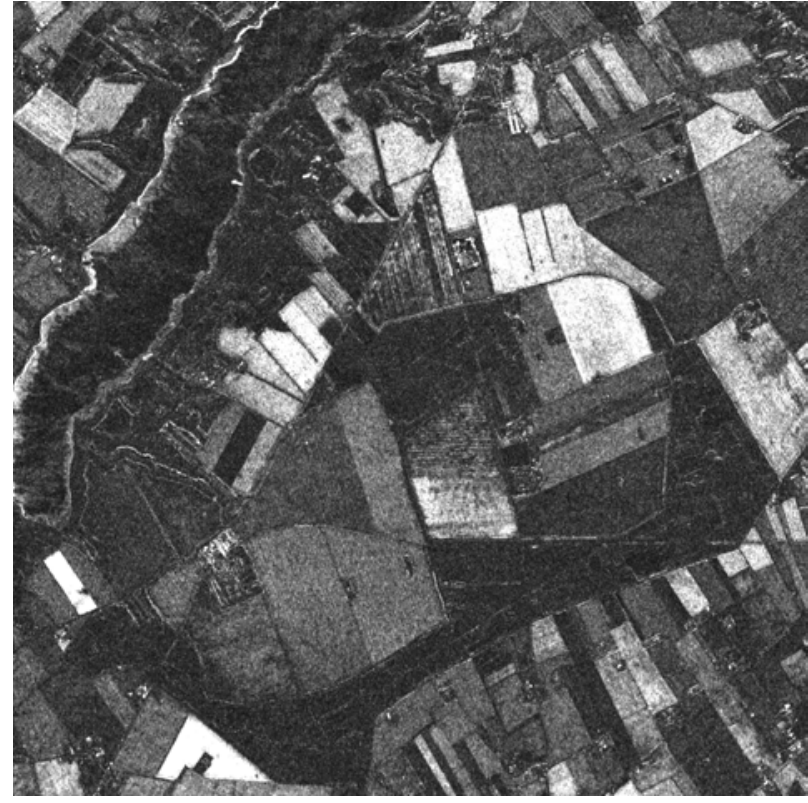

Fig. 9. $-2 \rho \ln Q$ for C64/L64 vs C65/L65, full polarimetry.

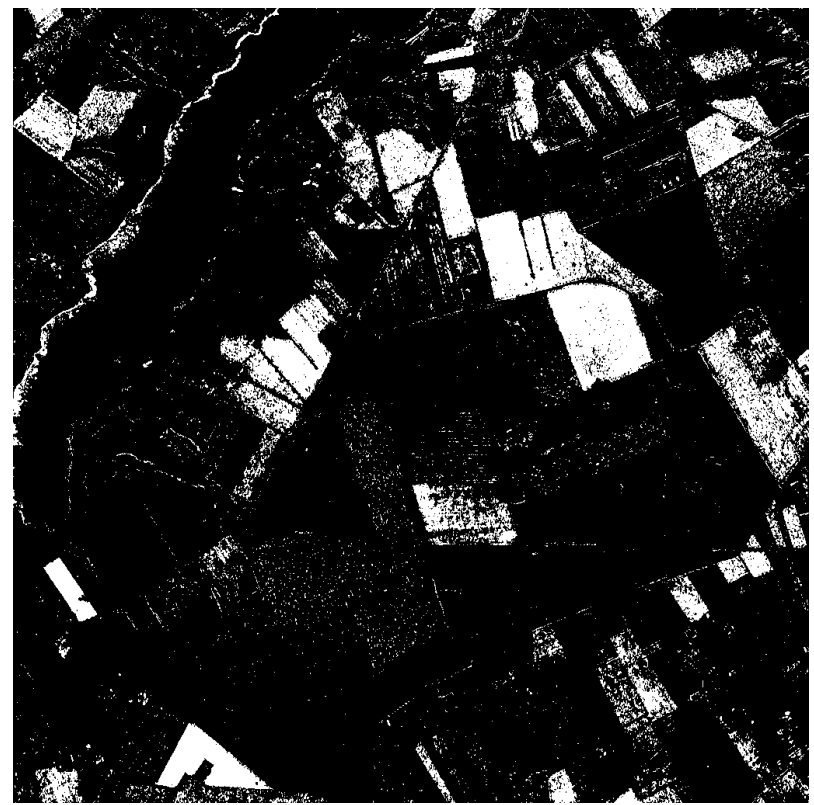

Fig. 10. $P\{-2 \rho \ln Q \leq z\}$ thresholded at 0.9999 for C64/L64 vs C65/L65, full polarimetry.

Figures 11 and 12 show $-2 \rho \ln Q$ (stretched between 0 and 50) and $P$ thresholded at 0.9999 for dual polarimetry L64 vs L65, HH/HV.

\section{DISCUSSION}

Wooded areas surrounding some of the large fields in the middle of the images and along the lake and which exhibit no change between the two acquisitions, are seen in cyan in Figures 1, 2, 3 and 4. Tables I and II show average backscatter coefficients, correlation and phase difference between $\mathrm{HH}$ and VV over a small wooded area and the beets field (see Figures 6 and 8) for C- and L-band data. Also they show the average

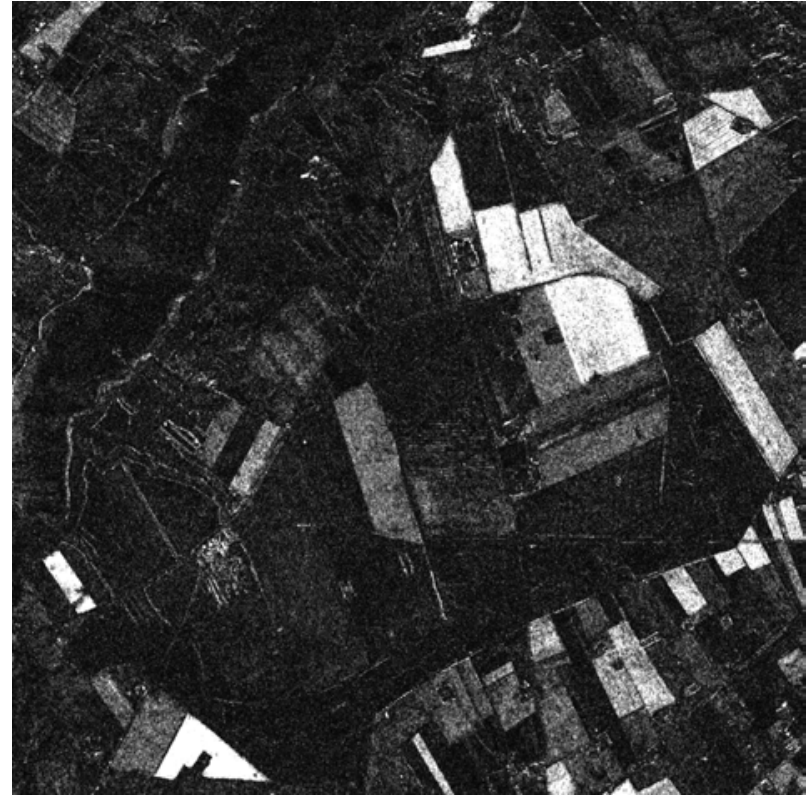

Fig. 11. $-2 \rho \ln Q$ for L64 vs L65, dual polarimetry HH/HV.

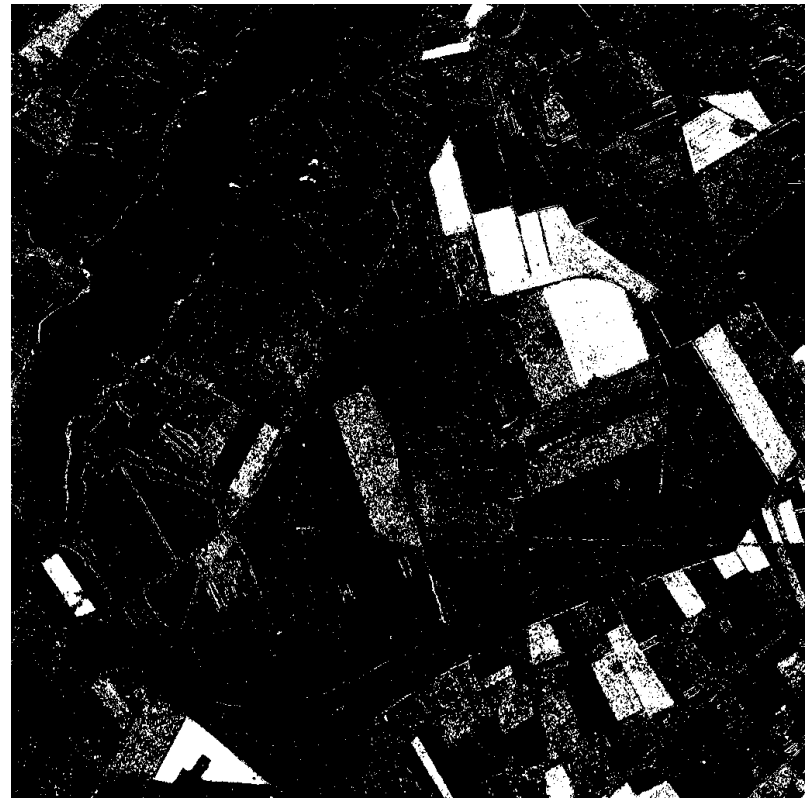

Fig. 12. $P\{-2 \rho \ln Q \leq z\}$ thresholded at 0.9999 for L64 vs L65, dual polarimetry $\mathrm{HH} / \mathrm{HV}$.

no-change probability associated with the test statistic. For the bi-frequency analysis $P\left\{Q<q_{\text {obs }}\right\}$ is 0.4893 for the wooded area and 0.0005 for the beets field. The wooded areas are all coniferous forests, and hence no change is occurring between May and June, in correspondence with the results of the change detector at all frequency combinations. The beets field, on the other hand, is changing from a bare field in May to a field with some leaves developed in June, and hence, changes are detected at all frequency combinations.

Changes are detected in the winter barley fields between May and June for C-band and the C- and L-band combinations, but not for the L-band alone. From May to June, the head 
TABLE I

AVERAGE POLARIMETRIC PARAMETERS AND NO-CHANGE PROBABILITY OVER A WOODED AREA FOR C-AND L-BAND DATA.

\begin{tabular}{|l|r|r|r|r|}
\hline & C May & C Jun & L May & L Jun \\
\hline$\sigma_{h h}^{0}[\mathrm{~dB}]$ & -6.5 & -6.1 & -5.4 & -5.4 \\
\hline$\sigma_{h v}^{0}[\mathrm{~dB}]$ & -12.7 & -12.2 & -10.6 & -10.6 \\
\hline$\sigma_{v v}^{0}[\mathrm{~dB}]$ & -7.2 & -6.7 & -7.1 & -7.3 \\
\hline$\rho$ & 0.49 & 0.48 & 0.38 & 0.41 \\
\hline$\phi_{h h-v v}[\mathrm{rad}]$ & -0.16 & -0.08 & -0.11 & -0.17 \\
\hline$P\left\{Q<q_{\text {obs }}\right\}$ & & 0.4850 & & 0.4913 \\
\hline
\end{tabular}

TABLE II

AVERAGE POLARIMETRIC PARAMETERS AND NO-CHANGE PROBABILITY OVER THE BEETS FIELD FOR C- AND L-BAND DATA.

\begin{tabular}{|l|r|r|r|r|}
\hline & C May & C Jun & L May & L Jun \\
\hline$\sigma_{h h}^{0}[\mathrm{~dB}]$ & -16.1 & -10.3 & -24.6 & -13.1 \\
\hline$\sigma_{h v}^{0}[\mathrm{~dB}]$ & -27.4 & -17.4 & -35.0 & -24.6 \\
\hline$\sigma_{v v}^{0}[\mathrm{~dB}]$ & -16.1 & -11.2 & -22.4 & -15.3 \\
\hline$\rho$ & 0.81 & 0.54 & 0.80 & 0.57 \\
\hline$\phi_{h h-v v}[\mathrm{rad}]$ & 0.21 & 0.12 & 0.40 & 1.11 \\
\hline$P\left\{Q<q_{\text {obs }}\right\}$ & & 0.0015 & & 0.0004 \\
\hline
\end{tabular}

of the winter barley starts to hang down, and hence due to such an angle of the head and size of the head relative to the wavelength at $\mathrm{C}$-band it generates cross-polarized backscatter in June [14]. This change in the backscatter causes a detected change from May to June in C-band, whereas the size of the head is small compared to the wavelength at L-band, and hence, it does not generate a change in the backscatter at Lband.

The peas fields develop from a stage of some leaves developed in May with a height of approximately $15 \mathrm{~cm}$ to a stage of emergence in June with heights from $40-60 \mathrm{~cm}$. At C-band, the backscatter is dominated by volume scattering at both acquisitions, and hence no changes are seen in the backscatter from the fields. The large change in biomass between the two acquisitions causes a change in the L-band backscatter, and a change is detected at L-band and also for the combination of C- and L-band.

The grass field shows a detected change from May to June at C-band, and also in the combination of C- and L-band. The grass changes from standing relatively straight up in May, to lying down in June. This change causes an increase in the cross-polarized backscatter at C-band, and hence the detection of the change at C-band. At L-band, on the other hand, the change is not as dramatic, because the L-band backscatter is more sensitive to the total biomass, which is not changing so much. At L-band, the backscatter in May includes some double-bounce scattering, whereas this component is smaller in June, where the grass is more randomly oriented. Some change is detected using full polarimetry at L-band as seen in Figure 8, whereas no change is detected using dual polarimetry $(\mathrm{HH} / \mathrm{HV})$, which is not sensitive to the doublebounce scattering.

It is clear from the above results, that the combination of C- and L-band for the change detection, captures all changes that are detected by either C- or L-band.

\section{SOFTWARE}

Six Matlab programs will be made available from the first author's homepage

- freadenvisar,

- rho_hhvv,

- wishart_det,

- wishart_change,

- wishart_change_dual4 and

- wishart_change_full6.

freadenvisar reads covariance matrix polarimetric SAR data (with an ENVI-like header file) from disk and stores them in the order needed for the change detection programs.

rho_hhvv calculates the (complex) correlation including the phase difference between $\mathrm{HH}$ and $\mathrm{VV}$ polarizations in the following cases

- full polarimetry (including azimuthal symmetry), and

- dual polarimetry.

wishart_det calculates determinants of complex variance-covariance matrices and flags singularity in the following cases

- full polarimetry (including azimuthal symmetry and diagonal only), and

- dual polarimetry (including diagonal only).

wishart_change tests for equality of two complex variance-covariance matrices in the following cases

- full polarimetry (including azimuthal symmetry and diagonal only),

- dual polarimetry (including diagonal only), and

- single channel only.

wishart_change_dual 4 tests for equality of two block diagonal complex variance-covariance matrices in the dual polarimetry case (it allows two dual polarimetry blocks only and is meant for multi-frequency applications, see Sections II-C3 and III-A).

wishart_change_full 6 tests for equality of two block diagonal complex variance-covariance matrices in the full polarimetry case (it allows two full polarimetry blocks only and is meant for multi-frequency applications, see Sections II-C3 and III-B).

The single frequency methods described in this paper are mentioned in [15] which also has software in ENVI/IDL and Python for the analysis.

\section{Conclusions}

Change in winter barley fields and in a grass field is detected in C-band and not in L-band. Change in pea fields is detected in L-band and not in C-band. The multi-frequency analysis detects change in both winter barley, the grass field, and the pea fields. Change in a beets field is detected in both C- and L-band as well as in the multi-frequency analysis.

The results obtained with the change detector are confirmed by the change and absence of change in the polarimetric parameters over the beets field and a wooded area, respectively.

Ongoing work includes extension of Wishart distribution based change analysis to the truly multi-temporal case. 


\section{REFERENCES}

[1] K. Conradsen, A. A. Nielsen, J. Schou, and H. Skriver, "A test statistic in the complex Wishart distribution and its application to change detection in polarimetric SAR data," IEEE Transactions on Geoscience and Remote Sensing, vol. 41, no. 1, pp. 4-19, Jan. 2003, http://www.imm.dtu.dk/pubdb/p.php?1219.

[2] J. Schou, H. Skriver, A. A. Nielsen, and K. Conradsen, "CFAR edge detector for polarimetric SAR images," IEEE Transactions on Geoscience and Remote Sensing, vol. 41, no. 1, pp. 20-32, Jan. 2003 , http://www.imm.dtu.dk/pubdb/p.php?1224.

[3] V. Akbari, S. N. Anfinsen, A. P. Doulgeris, and T. Eltoft, "The HotellingLawley trace statistic for change detection in polarimetric SAR data under the complex Wishart distribution," in IEEE IGARSS, Melbourne, Victoria, Australia, 21-26 July 2013, pp. 4162-4165.

[4] J. J. van Zyl and F. T. Ulaby, "Scattering matrix representation for simple targets," in Radar Polarimetry for Geoscience Applications, F. T. Ulaby and C. Elachi, Eds. Artech, Norwood, MA, 1990.

[5] S. V. Nghiem, S. H. Yueh, R. Kwok, and F. K. Li, "Symmetry properties in polarimetric remote sensing," Radio Science, vol. 27, no. 5, pp. 693 $711,1992$.

[6] T. W. Anderson, An Introduction to Multivariate Statistical Analysis, John Wiley, New York, third edition, 2003.

[7] R. Touzi, A. Lopes, and P. Bousquez, "A statistical and geometrical edge detector for SAR images," IEEE Transactions on Geoscience and Remote Sensing, vol. 26, no. 6, pp. 764-773, Nov. 1988.

[8] A. Lopes, E. Nezry, R. Touzi, and H. Laur, "Structure detection and statistical adaptive speckle filtering in SAR images," International Journal of Remote Sensing, vol. 13, no. 9, pp. 1735-1758, 1993.

[9] O. A. Pedersen, "Kortlægning ved hjælp af radarbilleder," M.S. thesis, Technical University of Denmark, 2007, in Danish.

[10] M. Abramowitz and I. A. Stegun, Handbook of Mathematical Functions, National Bureau of Standards, 1964.

[11] W. H. Press, B. P. Flannery, S. A. Teukolsky, and W. T. Vetterling, Numerical Recipes in C: The Art of Scientific Computing, Cambridge University Press, second edition, 1992.

[12] S. N. Madsen, E. L. Christensen, N. Skou, and J. Dall, "The Danish SAR system: Design and initial tests," IEEE Transactions on Geoscience and Remote Sensing, vol. 29, pp. 417-476, 1991.

[13] E. L. Christensen, N. Skou, J. Dall, K. Woelders, J. H. Jørgensen, J. Granholm, and S. N. Madsen, "EMISAR: An absolutely calibrated polarimetric L- and C-band SAR," IEEE Transactions on Geoscience and Remote Sensing, vol. 36, pp. 1852-1865, 1998.

[14] H. Skriver, M. T. Svendsen, and A. G. Thomsen, "Multitemporal Land C-band polarimetric signatures of crops," IEEE Transactions on Geoscience and Remote Sensing, vol. 37, pp. 2413-2429, 1999.

[15] M. J. Canty, Image Analysis, Classification and Change Detection in Remote Sensing. With Algorithms for ENVI/IDL and Python, Taylor \& Francis, CRC Press, third edition, 2014.

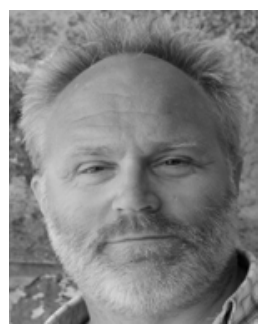

Allan Aasbjerg Nielsen received the M.Sc. degree from the Department of Electrophysics at the Technical University of Denmark (DTU), Lyngby, Denmark, in 1978 and the Ph.D. degree from Informatics and Mathematical Modelling (IMM), DTU, in 1994. $\mathrm{He}$ is currently an Associate Professor with the Department of Applied Mathematics and Computer Science, DTU. From 1977 to 1978, he was with the Danish Defense Research Establishment. From 1978 to 1985 , he worked on energy conservation in housing with the Thermal Insulation Laboratory, DTU. He was with the section for image analysis from 1985 to 2001 and with the section for geoinformatics from 2001 to 2006, both at IMM. From 2007 to 2013, he was with the Danish National Space Center's section for geodesy. Since 1985, he has worked on several national and international projects on the development, implementation, and application of statistical methods, and remote sensing in mineral exploration, mapping, geology, agriculture, environmental monitoring, oceanography, geodesy, and security funded by industry, the European Union, Danida (the Danish International Development Agency), and the Danish National Research Councils.

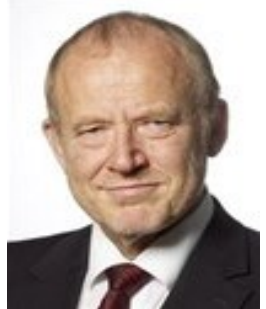

Knut Conradsen received the Cand. Scient. degree in mathematics in 1970 from the University of Copenhagen, Denmark. He has been with the Technical University of Denmark (DTU) since 1970. Presently he is Professor of Statistical Image Analysis at the Department of Applied Mathematics and Computer Science, DTU. From 1995 to 2010 he was Provost (Deputy Rector) of DTU. His main research interest is the application of statistics and statistical models in primarily medical image analysis, remote sensing and industrial applications. Work includes analysis of multi-/hyperspectral and multi-temporal data, as well from optical as from radar sensors.

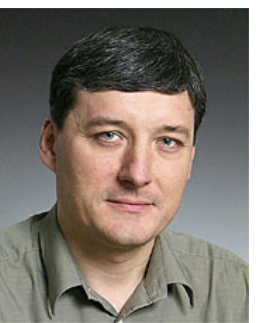

Henning Skriver received the M.Sc. and Ph.D. degrees in electrical engineering from the Technical University of Denmark (DTU), Lyngby, Denmark, in 1983 and 1989, respectively. Since 1983, he has been with the DTU, where he is an Associate Professor, the Head of Microwaves and Remote Sensing, and Deputy Head of Department at DTU Space. His work has primarily been concerned with topics related to the utilization of synthetic aperture radar (SAR) data for different applications, such as sea ice parameter retrieval from SAR data, as well as different aspects of land applications of SAR data, such as forestry, agricultural, environmental, and topographic mapping applications using both satellite SAR data and data from polarimetric SARs, e.g., the Danish airborne polarimetric SAR, EMISAR, and the German experimental SAR ESAR. His interests also include methods for the processing of SAR data; SAR image simulation; SAR image filtering; speckle statistics; and texture analysis, segmentation, calibration, change detection, classification, and polarimetric analysis and processing. 